\title{
Determining fitness to drive: A systematic review of the methods and assessments used after mild traumatic brain injury
}

\author{
Anne Baker ${ }^{1}$, Carolyn A Unsworth ${ }^{2,3,4,5}$ and Natasha A Lannin ${ }^{6,7}$
}

\begin{abstract}
Introduction: Limited evidence is available to support knowledge of the time-frame and capacity for fitness to drive after mild traumatic brain injury. The aim of this systematic review was to identify what methods and assessments are, or could be used to determine fitness to drive for this population.

Method: We undertook a systematic search of six electronic databases. Two authors rated all studies for methodological content and quality, and standardised data were extracted. Narrative analysis was conducted to understand the content of eligible studies. Findings: A total of 2022 articles were retrieved; seven articles met the inclusion criteria. Self-reported questionnaires, nonstandardised assessments, questionnaires completed by next-of-kin, and simulator tests were the primary methods used to determine fitness to drive. Only one assessment has been used to aid recommendations about fitness to drive in the acute hospital setting. Six additional standardised assessments were identified that have the potential to predict fitness to drive in this population group; however, these assessments require further psychometric testing prior to use.

Conclusion: While a variety of methods and assessments are currently used, there is little research evidence to suggest when individuals are able to return to driving after mild traumatic brain injury. Research is urgently required to determine a consistent and standardised approach to assessing fitness to drive following mild traumatic brain injury.
\end{abstract}

Keywords

Driving, driver assessment, fitness to drive, mild traumatic brain injury

Received: 16 December 2013; accepted: 5 August 2014

\section{Introduction}

Traumatic brain injury (TBI) refers to an injury that is sustained to the brain as a result of external forces. TBI severity can be classified as mild, moderate, or severe (McCrea et al., 2009). Of these three forms, it has been estimated that mild TBI (mTBI) occurs most commonly, and comprises $70-90 \%$ of all TBIs sustained in developed countries (Carroll et al., 2004). However, because a high proportion of individuals who sustain mTBI do not seek medical treatment, it is difficult to estimate the true prevalence and incidence of the condition. For those individuals who do seek medical treatment after mTBI, such treatment is most commonly provided in an acute hospital setting (Carroll et al., 2004). Mild TBI is characterised by an individual experiencing one or more of the following: confusion or disorientation after the event, the experience of other transient neurological abnormalities not requiring surgery, loss of consciousness (LOC) for $30 \mathrm{~min}-$ utes or less, post-traumatic amnesia (PTA) less than 24 hours, and a Glasgow Coma Scale (GCS) score of 13-15 up to 30 minutes post injury (Carroll et al., 2004). Patients may present with symptoms such as headache, dizziness, or fatigue post injury. In addition, patients who have sustained mTBI may display reduced attention and concentration levels, as well as impairments on standardised cognitive assessments in the areas of organisation, planning, and self-monitoring abilities (McCrea et al., 2009). Symptoms are most pronounced 24 hours after mTBI, rapidly improve within 1-2 weeks, and are usually fully resolved within 3 months post injury (McCrea et al., 2009). These symptoms have the potential to impact on an individual's ability to return to a range of occupations. For example, individuals who return to driving may be at an increased risk of motor vehicle accident involvement in the acute stages post injury. While the exact link between the

\footnotetext{
${ }^{1}$ Doctoral Candidate, La Trobe University, Bundoora, Victoria, Australia ${ }^{2}$ Professor, La Trobe University, Bundoora, Victoria, Australia

${ }^{3}$ Professor, Jönköping University, Jönköping, Sweden

${ }^{4}$ Professor, Curtin University, Bentley, Perth, Australia

${ }^{5}$ Professor, Central Queensland University, Melbourne, Victoria, Australia

${ }^{6}$ Associate Professor, La Trobe University, Bundoora, Victoria, Australia

${ }^{7}$ Associate Professor, Alfred Health, Prahran, Victoria, Australia

Corresponding author:

Carolyn A Unsworth, Professor, Central Queensland University, Department of Occupational Therapy, 120 Spencer Street, Melbourne, Victoria 3000, Australia.

Email: c.unsworth@cqu.edu.au
} 
effects of mTBI and road safety are not yet known, emerging evidence suggests that individuals with $\mathrm{mTBI}$ are significantly slower to respond to traffic hazards when compared with individuals with orthopaedic injuries at 24 hours post injury (Preece et al., 2010). In addition, it has been noted that a high proportion of individuals with $\mathrm{mTBI}$ are required to either adapt their driving or to develop strategies to compensate for self-perceived driving difficulties experienced up to 2-6 weeks post injury (Bottari et al., 2012; Sveen et al., 2013).

\section{Fitness to drive}

In developed countries, the car is the most common form of transportation (World Health Organization, 2013). However, driving is a complex task. An estimated 1.2 million people are killed worldwide in on-road crashes each year, and an additional 50 million people are seriously injured (World Health Organization, 2013). The World Health Organization has warned that these figures will continue to increase up to $65 \%$ over the next 20 years, unless there is plan to rapidly commit to the prevention of road trauma. Therefore, it is essential that individuals and their next-of-kin can be provided with evidence-based directives, inclusive of time-frames for successful return to driving after mTBI. Any suggested time-frames for return to driving must take into account the fact that individuals who have sustained mTBI may display reduced cognitive and physiological abilities (McCrea et al., 2009), and that deficits in such abilities have the potential to affect fitness to drive (Austroads, 2012). Together with medical staff, occupational therapists face the difficult task of assessing and making a recommendation as to whether a patient who has sustained mTBI is fit to drive. Drawing on research evidence may aid clinical reasoning in this area. Two systematic reviews have focused on fitness to drive as an outcome after TBI, and have included participants with mTBI (Classen et al., 2009; Ortoleva et al., 2012). Ortoleva et al. (2012) conducted a systematic review in order to identify the predictors of fitness to drive after a TBI of any level of severity, including mTBI. Seven studies met the inclusion criteria, five of which were identified by the authors as being of poor methodological quality. The authors concluded that there is "no sound basis at present for predicting driving capacity after traumatic brain injury' (Ortoleva et al., 2012: 302). Similar to this, Classen et al. (2009) sought to understand whether fitness to drive could be predicted after a TBI of any level of severity, including mTBI. While Classen et al. (2009) included 13 studies in this review, only three studies included participant/s with mTBI. For the mTBI population, there was no evidence of consistent predictors of fitness to drive post injury (Classen et al., 2009). Hence, occupational therapists in the acute care setting have very little evidence on which to base fitness to drive recommendations provided to their patients after mTBI. There is therefore a need to establish what methods and assessments are currently being used to guide fitness to drive recommendations in clinical practice, given that predictive factors alone are not valid or reliable (Classen et al., 2009; Ortoleva et al., 2012).

This systematic review was considered to add significantly to knowledge in this area, by examining the methods and assessments of fitness to drive that are used with this population group. This was chosen in favour of further exploration of predictors of fitness to drive, which have already found to be lacking in other reviews (Classen et al., 2009; Ortoleva et al., 2012). Considering the limited information available in the literature reviewed, it was also reasoned that it would be valuable to review what assessments could be used in this area of practice and included in future research. Specifically, these assessments needed to be suitable for use in the acute hospital setting, given that this is where the majority of individuals who sustain $\mathrm{mTBI}$ are treated and recommendations are made about return to driving by occupational therapists (Carroll et al., 2004).

\section{Aim}

The aims of this systematic review were to: (1) identify what methods are used to determine if individuals who have sustained mTBI are fit to drive post injury; (2) identify what assessments are used in the acute hospital setting to determine fitness to drive with this population group; and (3) identify standardised assessments that could be used in the acute hospital setting to predict fitness to drive after mTBI (including assessments that are available, but have not yet been used with a dedicated sample of mTBI participants). These aims were designed to inform occupational therapists of current best evidence concerning return to driving after $\mathrm{mTBI}$, and to direct further research into assessments that could be used in the acute care setting.

\section{Methods}

The full protocol for this systematic review is available from the corresponding author for this paper.

\section{Search strategy}

For each aim, the electronic databases MEDLINE, CINAHL, PsychInfo, Embase, The Cochrane Library, and OT Seeker were searched by one author (AB) from inception to 30 April 2013. The search strategy was first developed and used in MEDLINE, and was then adapted for use in each of the other electronic databases (Appendix). A broad search strategy was selected for use in order to identify as many relevant studies as possible. Therefore, the search did not include keywords for specific methods and assessments. Keywords mild traumatic brain injury; OR mild TBI; OR mild closed head injury; OR minor head injury; OR concussion; OR brain concussion; OR post concussion syndrome; OR acquired brain injury; OR ABI were combined with the keywords fit\$ to driv\$; OR automobile driv\$; OR driv\$ perform\$; OR driv\$ ability; OR driv\$ skills; OR driv\$ competence; OR driv\$. All keywords were mapped to 
Medical Subject Headings (MeSH) in each electronic database. All citations identified in the search of the electronic databases were downloaded into a bibliographic management software program (EndNote version X5), and duplicate studies were removed. Remaining studies were screened by two authors ( $\mathrm{AB}$ and $\mathrm{CU})$ to determine eligibility for inclusion in the review. If agreement could not be reached between the two authors after discussion of the article, the option to contact a third reviewer (NL) was available. If insufficient evidence was available from the title and abstract to make a decision on whether to include or exclude the article, the full text was obtained and screened. If further clarification was still subsequently required, the primary author of the article was contacted via email. The reference lists of all studies that met the inclusion criteria were screened to identify any other studies that were not initially identified by searching the electronic databases. Online citation tracking was also completed for all of the studies that met the inclusion criteria. The websites of key brain injury associations and road traffic associations were screened to identify any studies not published in journals.

\section{Inclusion/exclusion criteria: Aims 1 and 2}

For an article to be included in Aim 1 and Aim 2 of this systematic review, each of the following criteria had to be satisfied.

Population. Participants in the study had to:

- Have sustained mTBI, as defined by Carroll et al. (2004):

- confusion or disorientation after the event;

- the experience of transient neurological abnormalities not requiring surgery

- LOC $\leqslant 30$ minutes

- $\mathrm{PTA} \leqslant 24$ hours

- GCS score $13-15$

- Be free from any co-morbidities that may impact on fitness to drive, such as post-traumatic stress disorder

- Be a current inpatient at an acute hospital setting or have been admitted and discharged from an acute hospital setting, either to home or to a rehabilitation centre

Outcome. At least one outcome measure used in the study had to be related to fitness to drive; as characterised by one or more of the following:

- comprehensive driver evaluations, non-standardised assessments of fitness to drive, standardised assessments of fitness to drive, neuropsychological tests, off-road screening assessments, on-road assessments, information provision, self-reported questionnaires, questionnaires completed by next-of-kin, or computer-based driving simulator tests

Studies were excluded from this systematic review if one or more of the following factors were identified.
Population. Participant samples that were:

- Comprised of mixed diagnostic groups (e.g. mTBI and moderate and/or severe TBI) where data for the mTBI group was significantly different and could not be separated out from the other group $/ \mathrm{s}$, even once the primary author of the article had been contacted

- Comprised of positive imaging findings (e.g. cerebral abnormalities or lesions)

- Non-human participants (e.g. crash test dummies)

Study design. Types of studies that were:

- Opinion reviews, narrative reviews, or conference proceedings where no data could be extracted

- Non-full text studies

- Non-English studies

- Duplicate studies

These exclusion criteria were set in order to ensure that high-quality data pertaining to fitness to drive after mTBI were obtained.

\section{Inclusion/exclusion criteria: Aim 3}

For an assessment to be included in Aim 3 of this systematic review, each of the following criteria had to be satisfied. The assessment had to be:

- Standardised, and commercially available.

- Able to be administered in a busy, acute hospital setting. This meant that the assessment had to be: a stand-alone tool, suitable for bedside use, short in length (less than 15 minutes), able to be resumed if temporarily interrupted, and required no equipment to administer (other than the administration booklet, pen and paper).

- Related to on-road performance - as characterised by one or more of the following being recorded:

- 'pass' or 'fail' on an on-road test

- crash involvement (not self-reported)

- traffic violations (not self-reported)

- Published in at least two peer-reviewed publications, which reported on the psychometric properties of the tool.

Assessments were excluded if they were:

- Related to driving simulator-based outcomes, which required specialised hardware in addition to a standard computer.

\section{Quality assessment}

Quality assessment was independently completed by two authors (AB and CU) for all included studies. Two quality assessment tools were used: The Assessment of Multiple Systematic Reviews (AMSTAR) (Shea et al., 2007) and 
The Downs and Black Instrument (Downs and Black, 1998), which was modified for use in this systematic review.

The AMSTAR contains 11 items evaluating the methodological quality of a systematic review (Shea et al., 2007). The respondent answers either 'yes', 'no', 'can't answer', or 'not applicable' to each of the questions. One point is awarded for a 'yes' response. A score of 11 indicates high methodological quality in a systematic review, and a score of 0 indicates low methodological quality. The AMSTAR has been shown to have acceptable face and content validity (Shea et al., 2007).

The Downs and Black Instrument consists of 27 questions that are grouped into four sections: 'reporting', 'external validity', 'internal validity (bias)', and 'power' (Downs and Black, 1998). Scores on an unmodified Downs and Black Instrument range from 0-34, with a score of 0 indicating a low-quality study and a score of 34 indicating a high-quality study. The psychometric properties of the Downs and Black Instrument are reported to be acceptable for face and content validity, internal consistency (KR-20: 0.89), test-retest reliability ( $r 0.88)$, and inter-rater reliability ( $r$ 0.75) (Downs and Black, 1998). In addition, the Downs and Black Instrument can be adapted to suit the needs of a specific research question (Downs and Black, 1998). In this systematic review, all questions pertaining to 'intervention' (Question 4, 8, 14, 19, 23, 24) were removed from the instrument, as this review included a population (individuals who have sustained $\mathrm{mTBI}$ ) and outcome (fitness to drive) group only. Therefore the maximum score available on the Downs and Black Instrument in this systematic review was 21 .

All studies identified for inclusion in this systematic review were also graded on their level of evidence, as per The Centre for Evidence Based Medicine (2009). Using this system, each article was graded from Level 1a through to Level 5, with Level 1a offering the highest level of evidence and Level 5 offering the lowest level of evidence. This step was completed in order to allow studies that were assessed using the AMSTAR to be compared with studies that were assessed using The Downs and Black Instrument.

\section{Data extraction}

Data extraction was independently completed by two authors (AB and $\mathrm{CU}$ ) for all studies that met the inclusion criteria. For Aims 1 and 2, data from each article pertaining to: publication details, purpose of the study, study design, level of evidence provided by the study, and inclusion/exclusion criteria applied in the study were extracted. In addition, the population of the study, setting of the study, methods and assessments of fitness to drive used in the study, results of the study, conclusions from the study, and quality assessment of the study were extracted. For Aim 3, data were extracted about the assessments': commercial availability, time taken to complete, psychometric properties, relationship to on-road performance, and suitability to use with a population with $\mathrm{mTBI}$.

\section{Data analysis}

When planning this systematic review, it was anticipated that the search strategy would yield a wide variety of study designs, including systematic reviews, case-control studies, cohort studies, cross-sectional studies, longitudinal studies, and single case studies. Accordingly, it was anticipated that the methods and assessments of fitness to drive used in these studies would not be sufficiently homogenous to allow meaningful quantitative synthesis of the results. From the outset, it was therefore planned that data in this systematic review would be synthesised qualitatively, by using a narrative analysis for all three aims. This involved coding the data, and grouping them into like categories.

\section{Findings}

Figure 1 presents a flowchart of the number of studies that were identified and screened in this systematic review. Agreement was reached between the first two authors for all of the studies.

In summary, a total of 2022 studies were identified by applying the search strategy. After removing duplicate studies, 1063 studies remained. For Aims 1 and 2, seven studies met the inclusion criteria and were included in data analysis. Publication date of the included studies ranged from 1998 (Fisk et al., 1998) to 2010 (Preece et al., 2010). Except for one study, which was completed as a systematic review (Classen et al., 2009), all of the other studies used a cross-sectional design. The highest level of evidence of the studies was 1a, which was provided by the systematic review (Classen et al., 2009), with all other studies providing level $2 \mathrm{~b}$ evidence. A summary of each of the studies included in this systematic review is provided in Table 1. Two of the mTBI studies that were cited in the systematic review completed by Classen et al. (2009) were also identified for inclusion in this systematic review. Hence, in Table 1 the reader is referred to Study 3 (Hawley, 2001) and Study 7 (Schneider and Gouvier, 2005) for details of these studies. Furthermore, in the Results and Discussion presented hereafter, only six studies are listed, as the results from Classen et al. (2009) are contained either within Hawley (2001) or Schneider and Gouvier (2005).

\section{Primary aim}

What methods are used to determine if individuals who have sustained $\mathbf{m T B I}$ are fit to drive post injury? No consistency existed in the methods that were used in the studies included in this systematic review. Self-reported questionnaires were used in two of the studies (Fisk et al., 1998; Hawley, 2001). Non-standardised assessments of fitness to drive (Schneider and Gouvier, 2005), information provision (Moore and Leathem, 2004), questionnaires completed by next-of-kin (Kreutzer et al., 2009), and computer-based driving simulator tests (Preece et al., 2010) were used in one study each. Two studies (Moore 


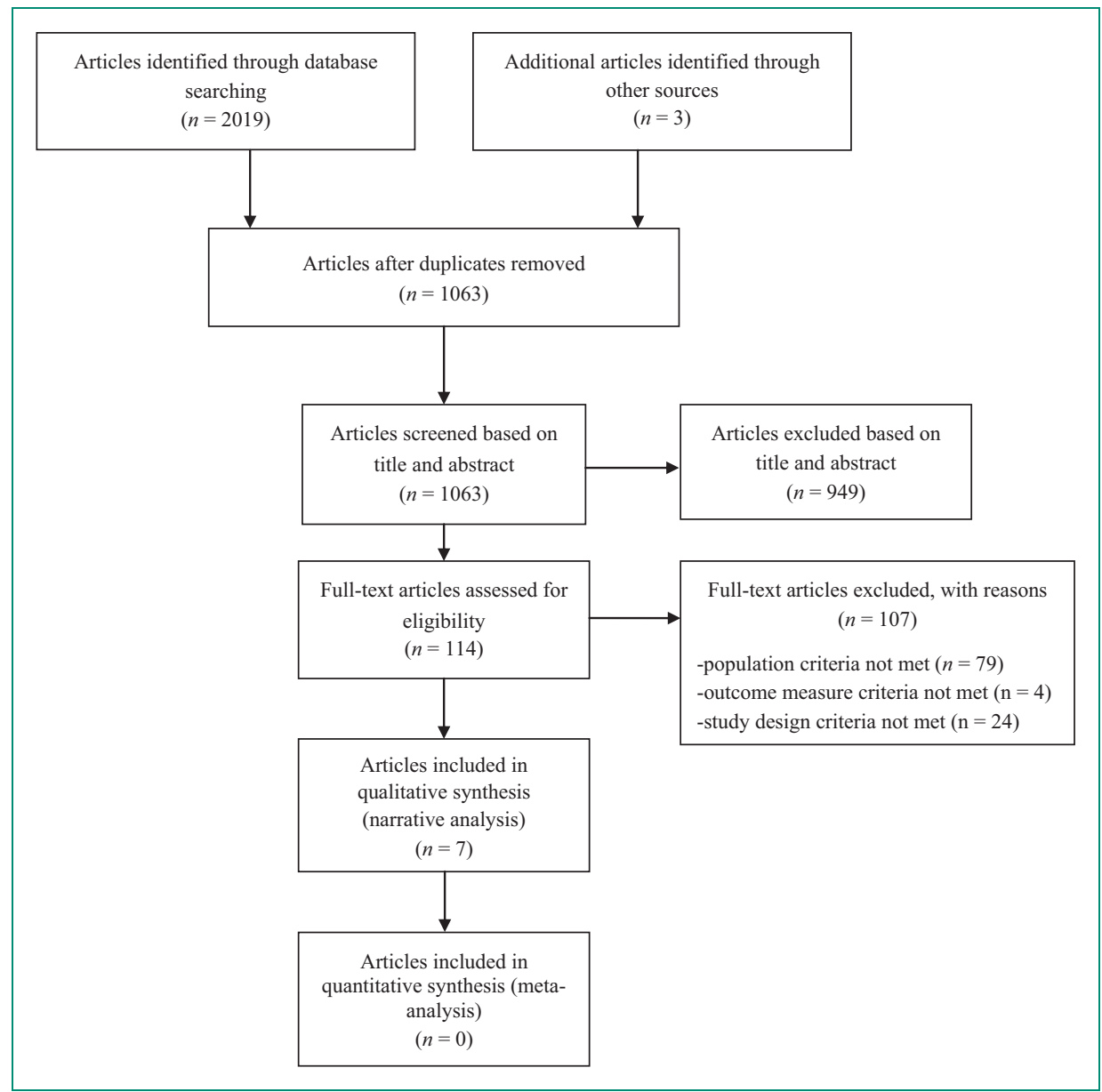

Figure 1. Number of studies identified and screened for inclusion or exclusion: Aim 1 and Aim 2.

and Leathem, 2004; Preece et al., 2010) were completed in the acute hospital setting. All of the other studies that were included in this systematic review used the method of referral to either inpatient rehabilitation or an outpatient setting to assess fitness to drive. This meant that time from injury to participation in the study varied, from an average of 24 hours post injury (Preece et al., 2010) to 7.13 years post injury (Schneider and Gouvier, 2005).

\section{Secondary aim}

What assessments are used specifically in the acute hospital setting to determine fitness to drive in individuals who have sustained mTBI? Two of the six studies included in this systematic review were completed in the acute hospital setting (Moore and Leathem, 2004; Preece et al., 2010). The study conducted by Moore and Leathem (2004) involved information provision to patients with mTBI about fitness to drive prior to discharge; however, it is not clear what assessment tool (if any) was used to inform these recommendations. In this study, blanket restrictions of 'no driving' for variable time periods (depending on the physician) were provided to all participants via an information handout, but it is not clear if actual assessment of fitness to drive did occur. Only one study included clear assessment of fitness to drive in the acute hospital setting (Preece et al., 2010). In this study, Preece et al. (2010) used The University of Queensland Hazard Perception Test (HPT) to examine the effects of mTBI on fitness to drive, 24 hours post injury. Convenience sampling was used to recruit 42 participants who had sustained mTBI, and 43 matched participants who had sustained an orthopaedic injury, from a large acute metropolitan hospital. On a computer screen, the HPT presented 24 traffic conflicts, and the participant was required to use a computer mouse to click on the traffic conflict as quickly as possible. It was found that participants who had sustained mTBI were significantly slower to respond to traffic hazards compared with participants who had sustained an orthopaedic injury ( $p=0.03, d=0.48)$. The authors concluded that individuals who have sustained mTBI should not drive for a minimum of 24 hours. These results are in line with the nature of recovery after $\mathrm{mTBI}$, where symptoms are most pronounced 24 hours post injury (McCrea et al., 2009), as well as clinical practice guidelines which advise patients not to 


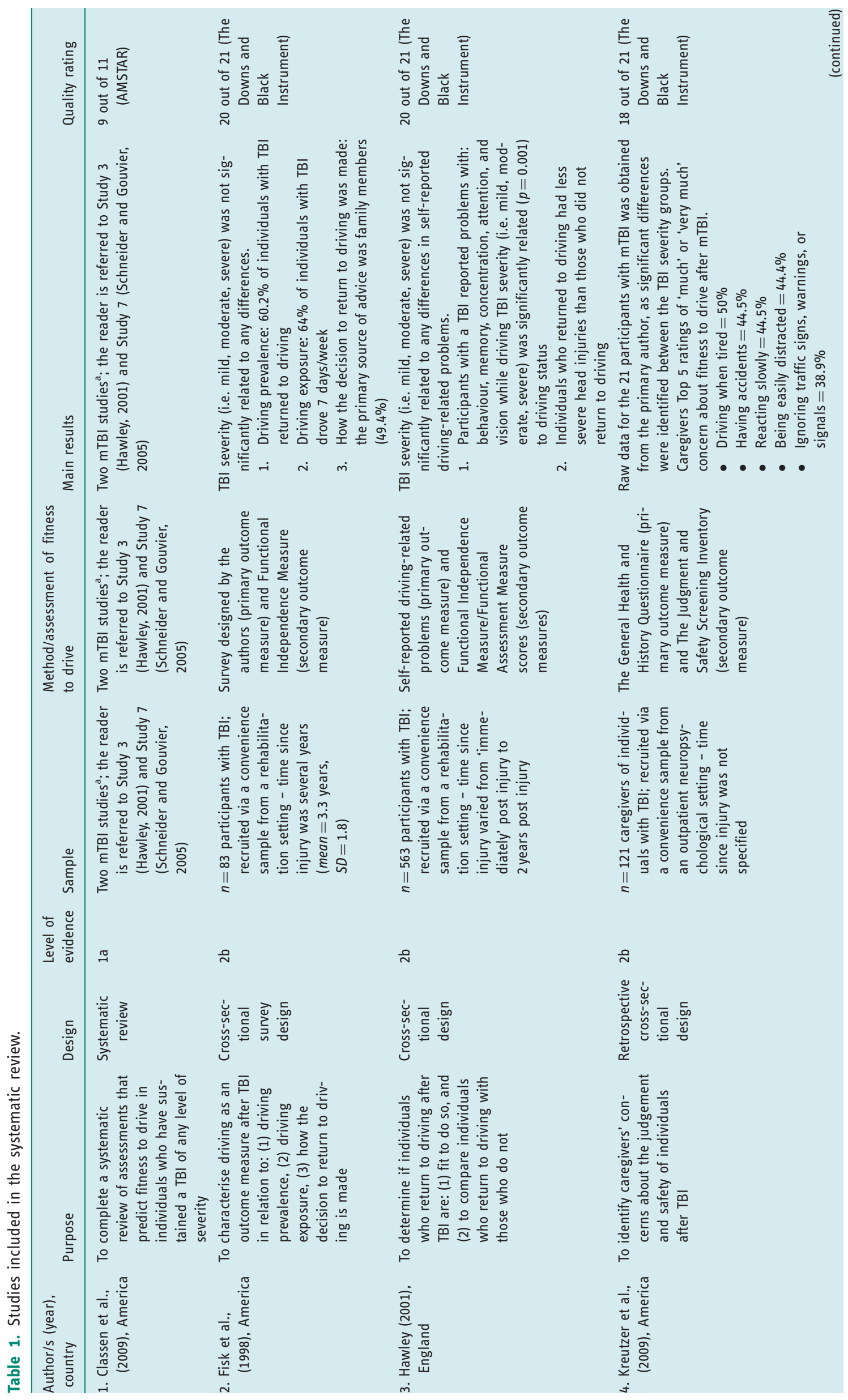




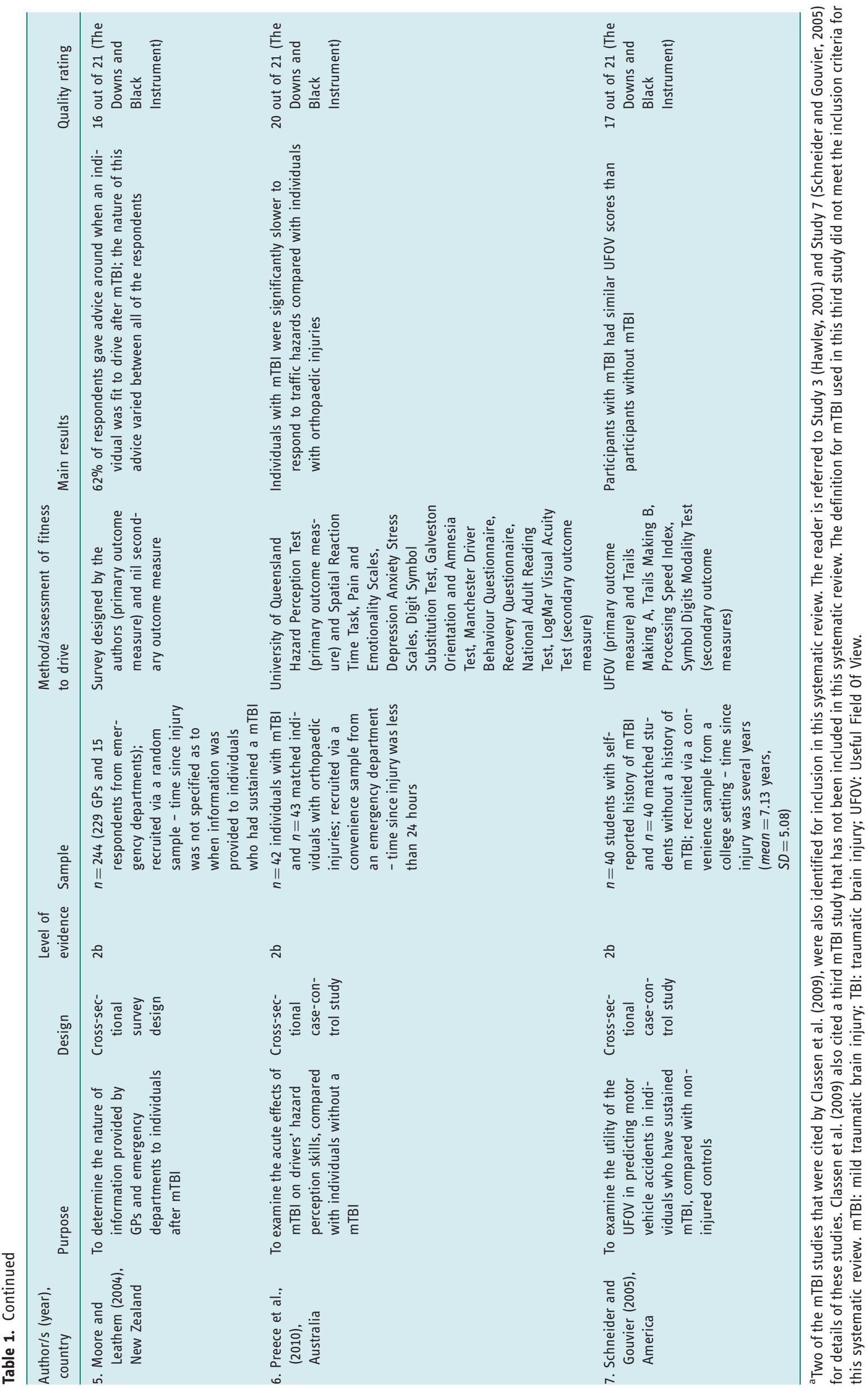


drive for a minimum of 24 hours (Austroads, 2012). This indicates that the University of Queensland HPT may be a valuable assessment of fitness to drive after mTBI, once psychometric studies with larger sample sizes are completed. However, as no longitudinal follow-up was provided in the study, it is not known at what time-point the participants were considered fit to drive after this 24 hour period. Findings suggest a need for future research to incorporate the expected recovery time-frame after mTBI into the methodology that is used.

\section{Tertiary aim}

What standardised assessments could be used in the acute hospital setting to predict fitness to drive after mTBI? In addition to the University of Queensland HPT which was identified through Aims 1 and 2 of this review, Aim 3 identified a further six standardised assessments that could be used to predict return to driving for this population group. A description of these assessments and their psychometric properties is presented in Table 2.

The Clock-Drawing Test (Shulman, 2000), MiniMental Status Exam (Folstein et al., 1975), OT Drive Home Maze Test (Unsworth et al., 2011a), Road Law and Road Craft Test (Unsworth et al., 2011b), Trail Making Test Part A (Reitan, 1986), and Trail Making Test Part B (Reitan, 1986) have all displayed the capacity to predict on-road driving performance in participants with moderate and severe TBI, and other forms of neurological conditions. However, as per Aim 1 and Aim 2 of this systematic review, no published studies currently exist to support fitness to drive recommendations with these six assessments after mTBI. Therefore, further psychometric testing for all of these assessments is indicated with this population group. These six assessments, as well as the HPT (Preece et al., 2010), are likely to offer occupational therapists with a selection of valid and reliable standardised assessments to aid their decision-making with respect to fitness to drive after $\mathrm{mTBI}$.

\section{Discussion}

This systematic review identified only seven studies pertaining to the methods and assessments used to determine whether individuals who have sustained mTBI are fit-todrive post injury. When planning this systematic review, it was decided to include studies where participants had been discharged from the acute hospital setting to either an inpatient rehabilitation setting or to outpatient followup. This was completed in order to provide an indication of the context of where fitness to drive is determined after mTBI. However, this meant that time from injury to participation in the study varied considerably, from 24 hours post injury to 7.13 years post injury. In line with this, five of the seven studies included in this systematic review were completed at either inpatient rehabilitation or at outpatient follow-up. This is surprising, given that individuals who sustain mTBI are most commonly treated in an acute hospital setting, and this is the context in which most clinical fitness to drive recommendations are made for this population group by occupational therapists (Carroll et al., 2004). In the two studies completed in the acute hospital setting, the methods of computer-based driving simulator testing (Preece et al., 2010) and information provision alone (Moore and Leathem, 2004) were used to determine fitness to drive recommendations. No studies used the 'gold standard' of Comprehensive Driver Evaluations (CDEs) (Classen et al., 2009) to assess fitness to drive after mTBI. Completed by specially trained occupational therapists, a CDE consists of an off-road assessment using tools that have been found to correlate with on-road driving performance, followed by an on-road assessment. For this population group, the off-road assessment could include the HPT or one or more of the six assessments identified in Aim 3: the Clock-Drawing Test (Shulman, 2000), Mini-Mental Status Exam (Folstein et al., 1975), OT Drive Home Maze Test (Unsworth et al., 2011a), Road Law and Road Craft Test (Unsworth et al., 2011b) Trail Making Test Part A (Reitan, 1986), and Trail Making Test Part B (Reitan, 1986), followed by an on-road assessment. The on-road assessment is completed in a dual-control vehicle with the individual presenting for assessment driving the car, the driving instructor sitting in the front passenger seat and controlling the car if necessary, and the occupational therapist sitting in the rear passenger seat. In this manner, an accurate and comprehensive assessment of the driver's performance can be obtained in the 'real world'; that is, on-road. However, CDEs are expensive to administer (Classen et al., 2009). This could be one reason why CDEs have not been used in research to date with participants who have sustained mTBI. However, if the expense of conducting a CDE with every patient who has sustained mTBI precludes its use in daily clinical practice, then use of the CDE in research with this population group is essential (Classen et al., 2009; Ortoleva et al., 2012), as CDEs offer the most valid and reliable form of fitness to drive assessment. Research using the CDE with a large sample of participants after mTBI could be used to establish evidence-based guidelines of off-road scores that predict appropriate time-frames for returning to drive post mTBI. Alternatively with further psychometric testing, the Clock-Drawing Test, Mini-Mental Status Exam, OT Drive Home Maze Test, Road Law and Road Craft Test, Trail Making Test Part A, and Trail Making Test Part B, or the HPT, could be used by occupational therapists in an acute hospital setting to screen patients for potential limitations in their fitness to drive. This would ensure that appropriate follow-up could be provided to these patients in an accurate and timely manner, and assist in preventing involvement in motor vehicle accidents.

\section{Limitations of the systematic review/directions for future research}

Although a rigorous search strategy was applied to this systematic review, it is possible that some studies that were 


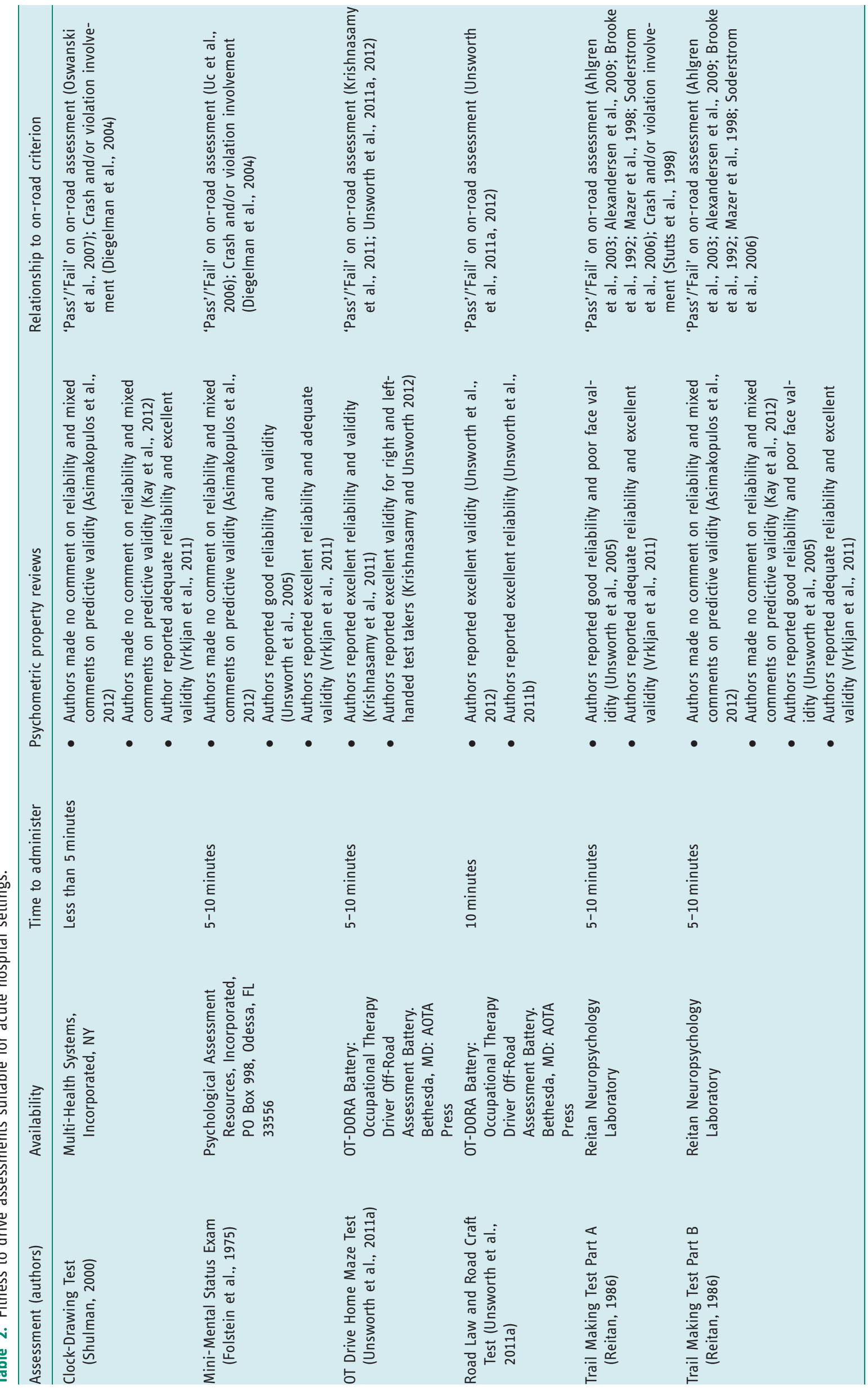


published in languages other than English were overlooked. In addition, because of the heterogeneity in the methods and assessments of fitness to drive used in each of the individual studies and the paucity of useful research data, it was not possible to pool the results using quantitative methods. However, this systematic review has highlighted a clear gap and a scarcity of published studies in the literature with respect to the methods and assessments of fitness to drive after mTBI. In particular, this review has identified that only two studies have been conducted in the acute hospital setting. Given that this is where the majority of individuals who sustain mTBI are treated (Carroll et al., 2004), further assessment of fitness to drive in the acute hospital setting is required. In addition, longitudinal follow-up of participants also needs to be incorporated into further research in this area. This will enable a trajectory of the expected recovery in fitness to drive skills after $\mathrm{mTBI}$ to be established. This research is urgently needed in order to reduce the risk of motor vehicle accidents and ensure the safety of all road users. Ultimately, the findings of this review and research in this area will promote the development of protocols to guide decision-making concerning fitness to drive post $\mathrm{mTBI}$.

\section{Conclusion}

In summary, this systematic review has demonstrated limited evidence concerning the methods and assessments that are used to determine if individuals who have sustained mTBI are fit to drive post injury. Since individuals who experience $\mathrm{mTBI}$ are generally expected to resume all of their pre-morbid occupations, it is essential that timeframes for the recovery of these skills are known. While there is growing research evidence for return-to-driving outcomes for patients who have moderate and even severe TBI, there is a paucity of information for the much larger group of patients with mTBI. One study has suggested that individuals should not drive for a minimum of 24 hours post mTBI. However, there is no evidence to support time-frames beyond this, and it is possible that patients who return to drive at 24 hours are still at risk of crash. Research is urgently required to provide occupational therapists with evidence-based directives, inclusive of time-frames, for successful return to driving with this population group. This kind of evidence will be of value not only to patients, but also to their families, clinicians, insurers, and third party payers. Ultimately, this research will play a role in maintaining a safer environment for all road users. In the interim, this review has identified seven standardised assessment that occupational therapists can use in acute care hospital settings to help guide clinical recommendations made to $\mathrm{mTBI}$ patients regarding their readiness to resume driving.

\section{Key findings}

- No consistency existed in the methods and assessments used with this population.
- Six standardised assessments were identified that could be used by occupational therapists to potentially determine fitness to drive with the mTBI population.

\section{What the study has added}

This systematic review presents current best evidence surrounding fitness to drive methods and assessments after $\mathrm{mTBI}$. The findings promote further research into assessments that could be used with this population group.

\section{Acknowledgements}

The authors would like to acknowledge Dr Tania Pizzari for her advice when planning this systematic review.

\section{Ethics}

Ethics approval was not required for this study.

\section{Declaration of conflicting interests}

None declared.

\section{Funding}

This research received no specific grant support from any funding agency in the public, commercial, or not-for-profit sectors.

\section{References}

Ahlgren E, Lundqvist A, Nordlund A, et al. (2003) Neurocognitive impairment and driving performance after coronary artery bypass surgery. European Journal of CardioThoracic Surgery 23(3): 334-340.

Alexandersen A, Dalen K and Bronnick K (2009) Prediction of driving ability after inconclusive neuropsychological investigation. Brain Injury 23(4): 313-321.

Asimakopulos J, BoychuckZ, Sondergaard D, et al. (2012) Assessing executive function in relation to fitness to drive: A review of tools and their ability to predict safe driving. Australian Occupational Therapy Journal 59(5): 402-427.

Austroads (2012) Assessing fitness to drive: For commercial and private vehicle drivers. Sydney: Austroads.

Bottari C, Lamothe MP, Gosselin N, et al. (2012) Driving difficulties and adaptive strategies: The perception of individuals having sustained a mild traumatic brain injury. Rehabilitation Research and Practice 2012: 9.

Brooke MM, Questad KA, Patterson DR, et al. (1992) Driving evaluation after traumatic brain injury. American Journal of Physical Medicine and Rehabilitation 71(3): 177-182.

Carroll LJ, Cassidy JD, Holm L, et al. (2004) Methodological issues and research recommendations for mild traumatic brain injury: The WHO collaborating centre task force on mild traumatic brain injury. Journal of Rehabilitation Medicine 43(Supplement): 113-125.

Centre for Evidence Based Medicine (2009) OCEBM levels of evidence. Available at: $h t t p: / / w w w . c e b m . n e t / ? o=1025$ (accessed 1 December 2013).

Classen S, Levy C, McCarthy D, et al. (2009) Traumatic brain injury and driving assessment: An evidence-based literature review. American Journal of Occupational Therapy 64(5): 580-591.

Diegelman NM, Gilbertson AD, Moore JL, et al. (2004) Validity of the clock-drawing test in predicting reports of driving problems in the elderly. BMC Geriatrics 4: 10-16. 
Downs SH and Black N (1998) The feasibility of creating a checklist for the assessment of the methodological quality both of randomised and non-randomised studies of health care interventions. Journal of Epidemiology and Community Health 52(6): 377-384.

Fisk GD, Schneider JJ and Novack TA (1998) Driving following traumatic brain injury: Prevalence, exposure, advice and evaluations. Brain Injury 12(8): 683-695.

Folstein MF, Folstein SE and McHugh PR (1975) Mini-mental state: A practical method for grading the cognitive state of patients for the clinician. Journal of Psychiatric Research 12: 189-198.

Hawley CA (2001) Return to driving after head injury. Journal of Neurology. Neurosurgery, and Psychiatry 70(6): 761-766.

Kay LG, Bundy AC, Clemson L, et al. (2012) Contribution of off-road tests to predicting on-road performance: A critical review of tests. Australian Occupational Therapy Journal 59(1): 89-97.

Kreutzer JS, Livingston LA, Everley RS, et al. (2009) Caregivers' concerns about judgement and safety of patients with brain injury: A preliminary investigation. Physical Medicine and Rehabilitation 1(8): 723-728.

Krishnasamy C and Unsworth CA (2011) Normative data, preliminary inter-rater reliability and predictive validity of the Drive Home Maze Test. Clinical Rehabilitation 25(1): 88-95.

Krishnasamy C and Unsworth CA (2012) Validity of the Occupational Therapy-Drive Home Maze Test for right and left handed test takers. Journal of Accident Analysis and Prevention 49: 274-277.

Mazer BL, Korner-Bitensky NA and Sofer S (1998) Predicting ability to drive after stroke. Archives of Physical Medicine and Rehabilitation 79(7): 743-750.

McCrea M, Iverson GL, McAllister TW, et al. (2009) An integrated review of recovery after mild traumatic brain injury (mTBI): Implications for clinical management. The Clinical Neuropsychologist 23(8): 1368-1390.

Moore C and Leathem J (2004) Information provision after mild traumatic brain injury (mTBI): A survey of general practitioners and hospitals in New Zealand. The New Zealand Medical Journal 117(1201): 1-9.

Ortoleva C, Brugger C, Van der Linden M, et al. (2012) Prediction of driving capacity after traumatic brain injury: A systematic review. Journal of Head Trauma Rehabilitation 27(4): 302-313.

Oswanski MF, Sharma OP, Raj SS, et al. (2007) Evaluation of two assessment tools in predicting driving ability of senior drivers. American Journal of Physical Medicine \& Rehabilitation 86(3): 190-199.

Preece MHW, Horswill MS and Geffen GM (2010) Driving after concussion: The acute effect of mild traumatic brain injury on drivers' hazard perception. Journal of Neuropsychology 24(4): 493-503.

Reitan RM (1986) Trail Making Test manual for administration and scoring. Tucson, AZ: Reitan Neuropsychology Laboratory.

Schneider JJ and Gouvier WD (2005) Utility of the UFOV test with mild traumatic brain injury. Applied Neuropsychology 12(3): $138-142$

Shulman KI (2000) Clock-drawing: Is it the ideal cognitive screening test? International Journal of Geriatric Psychiatry 15(6): 548-561.

Shea BJ, Grimshaw JM, Wells GA, et al. (2007) Development of AMSTAR: A measurement tool to assess the methodological quality of systematic reviews. BMC Medical Research Methodology 7(10): 1-7.

Soderstrom ST, Pettersson RP and Leppert J (2006) Prediction of driving ability after stroke and the effect of behind-the-wheel training. Scandinavian Journal of Psychology 47(5): 419-429.

Stutts JC, Stewart JR and Martell C (1998) Cognitive test performance and crash risk in an older driver population. Journal of Accident Analysis \& Prevention 30(3): 337-346.

Sveen U, Ostensjo S, Laxe S, et al. (2013) Problems in functioning after a mild traumatic brain injury within the ICF framework: The patient perspective using focus groups. Disability and Rehabilitation 35(9): 749-757.

Uc EY, Rizzo M, Anderson SW, et al. (2006) Driving with distraction in Parkinson disease. Neurology 67(10): 1774-1780.

Unsworth CA, Baker A, Taitz C, et al. (2012) Development of a standardised Occupational Therapy Driver Off-road Assessment (OT-DORA) battery to assess older and/or functionally impaired drivers. Australian Occupational Therapy Journal 59(1): 23-36.

Unsworth CA, Lovell RK, Terrington NS, et al. (2005) Review of tests contributing to the occupational therapy off-road driver assessment. Australian Occupational Therapy Journal 52(1): 57-74.

Unsworth CA, Pallant JP, Russell KJ, et al. (2011a) OT-DORA Battery: Occupational Therapy Driver Off-Road Assessment Battery. Bethesda, MD: AOTA Press.

Unsworth CA, Pallant JF, Russell K, et al. (2011b) Inter rater reliability of the Road Law and Road Craft test as part of the OT-DORA Battery for off-road driver assessment. British Journal of Occupational Therapy 74(8): 394-398.

Vrkljan BH, McGrath CE and Letts LJ (2011) Assessment tools for evaluating fitness to drive: A critical appraisal of evidence. Canadian Journal of Occupational Therapy 78(2): 80-96.

World Health Organization (2013) Global status report on road safety 2013. Geneva: WHO. Available at: http:// www.who.int/violence_injury_prevention/road_safety_status/ 2013/en/index.html (accessed 1 December 2013). 


\section{Appendix}

\section{MEDLINE search strategy}

Line

number Result

1

'mild traumatic brain injury'.mp. [ $\mathrm{mp}=$ title, abstract, original title, name of substance word, subject heading word, protocol supplementary concept, rare disease supplementary concept, unique identifier]

2 'mild TBI'.mp. [mp = title, abstract, original title, name of substance word, subject heading word, protocol supplementary concept, rare disease supplementary concept, unique identifier]

3 'mild closed head injury'.mp. [ $\mathrm{mp}=$ title, abstract, original title, name of substance word, subject heading word, protocol supplementary concept, rare disease supplementary concept, unique identifier]

4 'minor head injury'.mp. [mp = title, abstract, original title, name of substance word, subject heading word, protocol supplementary concept, rare disease supplementary concept, unique identifier]

5 'concussion'.mp. [mp = title, abstract, original title, name of substance word, subject heading word, protocol supplementary concept, rare disease supplementary concept, unique identifier]

6 'post-concussion syndrome'.mp. [ $\mathrm{mp}=$ title, abstract, original title, name of substance word, subject heading word, protocol supplementary concept, rare disease supplementary concept, unique identifier]

7 'brain concussion'.mp. [mp = title, abstract, original title, name of substance word, subject heading word, protocol supplementary concept, rare disease supplementary concept, unique identifier]

8 'acquired brain injury'.mp. [mp = title, abstract, original title, name of substance word, subject heading word, protocol supplementary concept, rare disease supplementary concept, unique identifier]

9 'ABI'.mp. [mp = title, abstract, original title, name of substance word, subject heading word, protocol supplementary concept, rare disease supplementary concept, unique identifier]

10 exp Post-Concussion Syndrome/ or exp Brain Injuries/ or exp Brain Concussion/ or exp Head Injuries, Closed/

$11 \quad 1$ or 2 or 3 or 4 or 5 or 6 or 7 or 8 or 9 or 10

12 'fit\$ to driv\$'.mp. [mp = title, abstract, original title, name of substance word, subject heading word, protocol supplementary concept, rare disease supplementary concept, unique identifier]

13 'automobiledriv\$'.mp. [mp = title, abstract, original title, name of substance word, subject heading word, protocol supplementary concept, rare disease supplementary concept, unique identifier]

14 'driv\$ perform\$'.mp. [ $\mathrm{mp}=$ title, abstract, original title, name of substance word, subject heading word, protocol supplementary concept, rare disease supplementary concept, unique identifier]

15 'driv\$ ability'.mp. [mp = title, abstract, original title, name of substance word, subject heading word, protocol supplementary concept, rare disease supplementary concept, unique identifier]

16 'driv\$ skill\$'.mp. [mp = title, abstract, original title, name of substance word, subject heading word, protocol supplementary concept, rare disease supplementary concept, unique identifier]

17 'driv\$ competence'.mp. [mp = title, abstract, original title, name of substance word, subject heading word, protocol supplementary concept, rare disease supplementary concept, unique identifier]

18 'driv\$'.mp. [mp = title, abstract, original title, name of substance word, subject heading word, protocol supplementary concept, rare disease supplementary concept, unique identifier]

19 exp Automobile Driving/ or exp Automobile Driver Examination/

2012 or 13 or 14 or 15 or 16 or 17 or 18 or 19

$21 \quad 11$ and 20

$22 \quad$ limit 21 to humans 